\title{
Figure Correction: Health Information Technology in Healthcare Quality and Patient Safety: Literature Review
}

Sue S Feldman ${ }^{1 *}$, RN, MEd, PhD; Scott Buchalter ${ }^{2 *}, \mathrm{MD}$; Leslie W Hayes ${ }^{3 *}$, MD

${ }^{1}$ Department of Health Services Administration, The University of Alabama at Birmingham, Birmingham, AL, United States

${ }^{2}$ Pulmonary and Critical Care, The University of Alabama at Birmingham Medical Center, Birmingham, AL, United States

${ }^{3}$ Department of Pediatrics, The University of Alabama at Birmingham Medical Center, Birmingham, AL, United States

*all authors contributed equally

\section{Corresponding Author:}

Sue S Feldman, RN, MEd, PhD

Department of Health Services Administration

The University of Alabama at Birmingham

1716 9th Avenue

SHPB \#590K

Birmingham, AL, 35294

United States

Phone: 16616188805

Email: suefeldman1009@gmail.com

\section{Related Article:}

Correction of: http://medinform.jmir.org/2018/2/e10264/

(JMIR Med Inform 2019;7(1):e11320) doi: 10.2196/11320

The authors of "Health Information Technology in Healthcare Quality and Patient Safety: Literature Review" (JMIR Med Inform 2018;6(2):e10264) mistakenly provided revised figures that were not identical in every way to the original ones in the paper that they were meant to replace.

During proofreading, the authors were asked to provide updated versions of Figures 4-7 because the versions that were originally provided were not of sufficient resolution. When creating better quality images, the authors accidentally omitted frequencies (groundedness and densities) that were included in the original images.

The incomplete versions of Figures 4-7 have been replaced and can be viewed below.

The correction will appear in the online version of the paper on the JMIR website on January 3, 2019, together with the publication of this correction notice. Because this was made after submission to PubMed, PubMed Central, and other full-text repositories, the corrected article also has been resubmitted to those repositories. 
Figure 4. ACTION Network Diagram ( $G=$ groundedness, $D=$ density). 


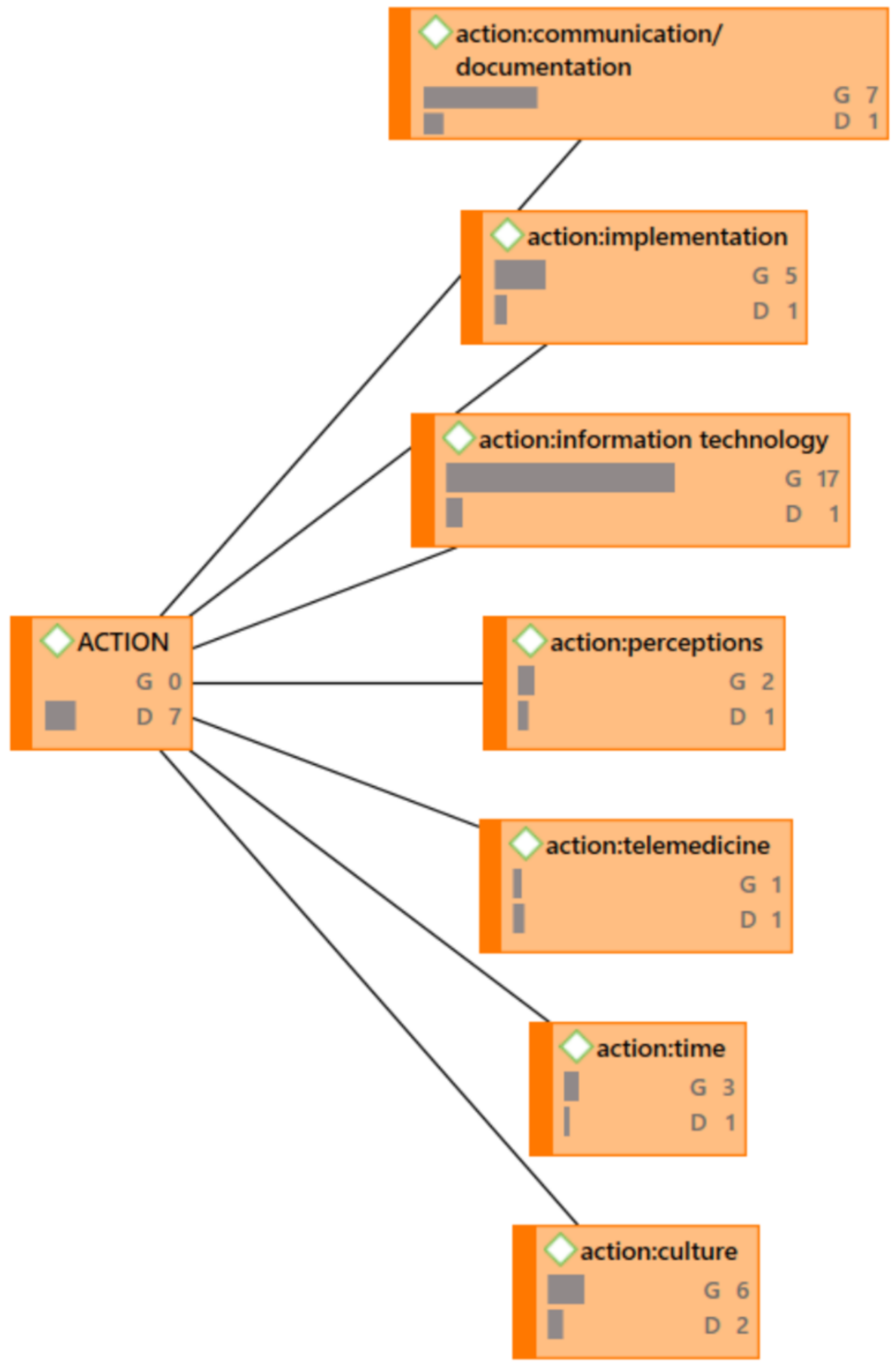


Figure 5. OUTCOMES Network Diagram ( $\mathrm{G}=$ groundedness, $\mathrm{D}=$ density)

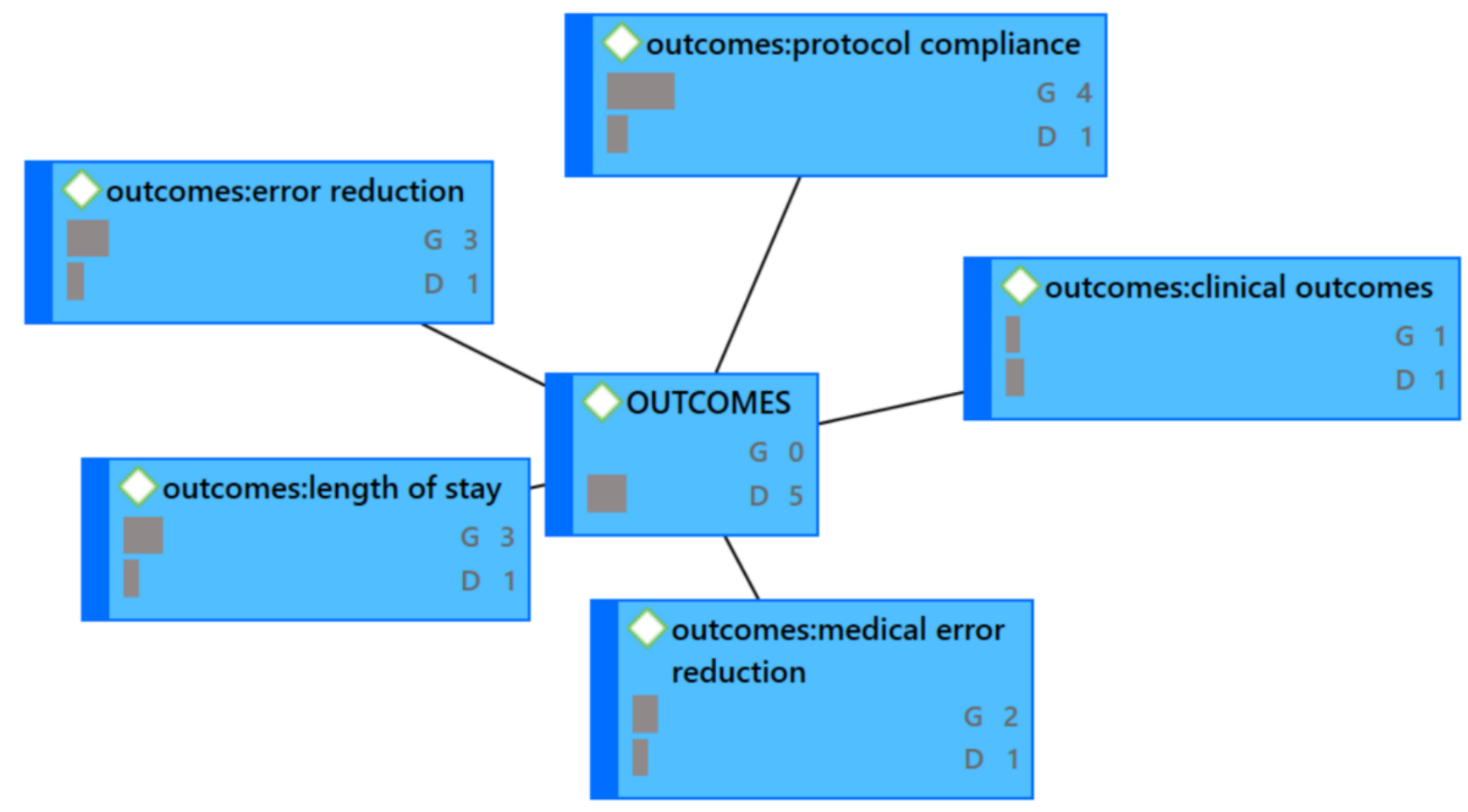


Figure 6. IDENTIFICATION Network Diagram (G=groundedness, $D=$ density).

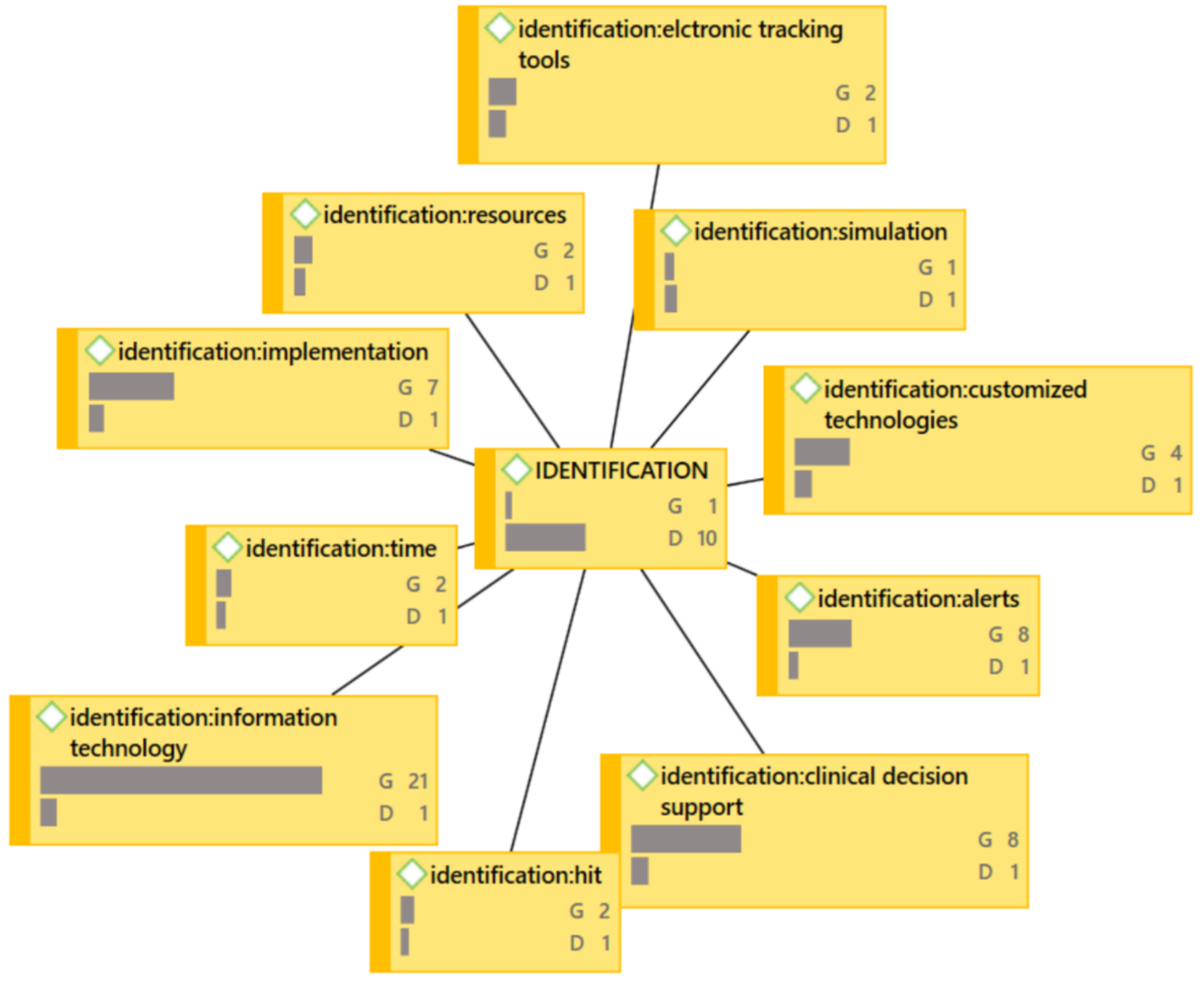


Figure 7. PREVENTION Network Diagram (G=groundedness, $D=$ density).

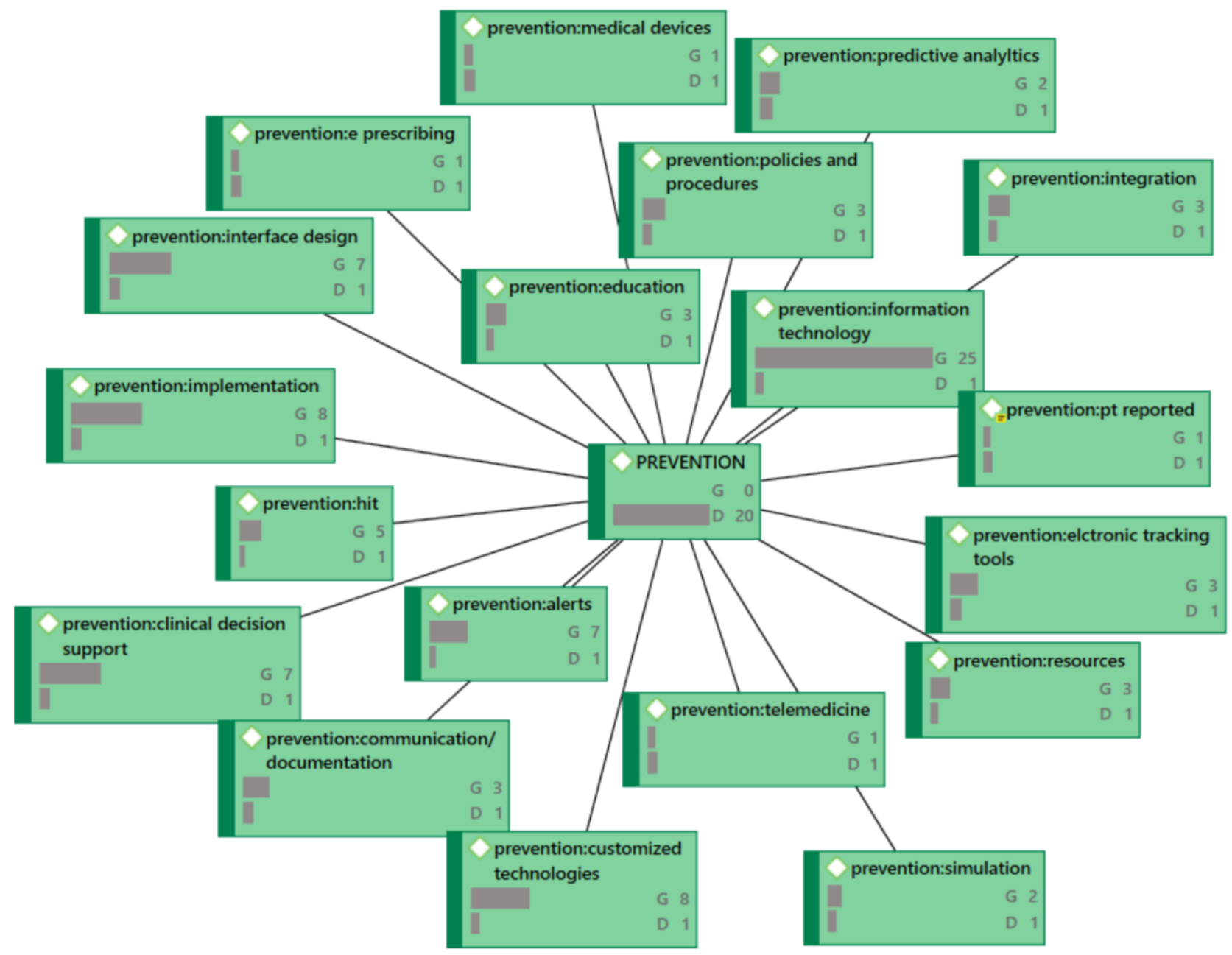

Edited by G Eysenbach; this is a non-peer-reviewed article. Submitted 18.06.18; accepted 09.10.18; published 03.01.19.

Please cite as:

Feldman SS, Buchalter S, Hayes LW

Figure Correction: Health Information Technology in Healthcare Quality and Patient Safety: Literature Review

JMIR Med Inform 2019;7(1):e11320

URL: https://medinform.jmir.org/2019/1/e11320/

doi: $10.2196 / 11320$

PMID: 30609984

(C) Sue S Feldman, Scott Buchalter, Leslie W. Hayes. Originally published in JMIR Medical Informatics (http://medinform.jmir.org), 03.01.2019. This is an open-access article distributed under the terms of the Creative Commons Attribution License (https://creativecommons.org/licenses/by/4.0/), which permits unrestricted use, distribution, and reproduction in any medium, provided the original work, first published in JMIR Medical Informatics, is properly cited. The complete bibliographic information, a link to the original publication on http://medinform.jmir.org/, as well as this copyright and license information must be included. 